ALFVEN FREQUENCY MODES AND GLOBAL ALFVEN EIGENMODES

L. Villard \& J. Vaclavik

submitted for publication to Nuclear Fusion 


\title{
Alfvén Frequency Modes and Global Alfvén Eigenmodes
}

\author{
L.Villard and J. Vaclavik \\ Centre de Recherches en Physique des Plasmas \\ Association Euratom - Confédération Suisse \\ Ecole Polytechnique Fédérale de Lausanne \\ 21, av. des Bains - CH-1007 Lausanne/Switzerland
}

\section{Abstract}

The spectrum of $n=0$ Alfvén modes is calculated analytically and numerically in cylindrical and toroidal geometries. It includes Global Alfvén Eigenmodes (GAE) and Surface Modes (SM) of the fast magnetoacoustic wave. These modes are not induced by toroidicity. The $n=0$ GAEs owe their existence to the shear. The frequency spacing between different radial and poloidal modes and the correlation of eigenfrequencies with changes in the edge density are examined and found in complete agreement with experimental observations of what has been named the "Alfvén Frequency Mode" (AFM) so far [1]. Although the eigenfrequency is related to the edge density, the $n=0 \mathrm{GAE}$ (AFM) is not necessarily edge-localized.

\section{Introduction}

Interest in Alfvén waves in the context of their possible destabilization by fast particles [2],[3] has been revived in the last decade since the discovery of the Toroidicity-induced Alfvén Eigenmode (TAE) [4] and its observation in several tokamak experiments [5]-[7] where it was destabilized by injected high-energy beam ions. The dangerous modes are those with the lowest possible frequency, because a necessary condition for instability is that the eigenfrequency must be smaller than the drift frequency of the fast ions. For stellarators the most dangerous modes are not TAEs but the "cylindrical" Global Alfvén Eigenmodes (GAE), because the low shear and large aspect ratio does not allow for the existence of TAEs. The GAEs have been shown to be driven unstable by injected beam 
ions in the W-7AS stellarator [8]. One should also mention the possibility to study low frequency Alfvén waves by exciting them with the saddle coils antennas installed in the JET tokamak [9],[10]. While interest has so far focused on the TAEs, we show in this paper that the GAEs can be as important and that they could be studied on JET as well.

In a recent paper [1] experimental observations made on the tokamak TFTR have been reported of a high frequency ( $200-400 \mathrm{kHz}$ ) mode that was detected on the magnetic fluctuation measurements. This mode has been observed under a wide variety of operational regimes in NBI heated plasmas and in some ohmic plasmas. It has the following characteristics:

1. Its frequency scales with the Alfvén velocity.

2. Its frequency is in the same range as - although consistently above - the expected TAE frequency.

3. Its frequency correlates with variations in the edge density. More precisely, it is related to the Alfvén frequency for $n=0, m=1$ near $r / a=0.9$.

4. The toroidal mode number is $n=0$ while the poloidal structure shows typically a standing wave structure with $m=1,2$ main poloidal components.

5. The mode has maximal amplitude where the plasma touches the limiter ("antiballooning" if it is the inside limiter, "ballooning" if it is the outside limiter).

6. There are one or two modes, separated in frequency by about $25 \%$ (Fig.2 of Ref. [1]), or in some cases at twice the frequency (Fig.11 of [1], end of the discharge).

We intend to give a theoretical explanation to these observations. We do not address the question of an excitation mechanism, nor of the non-linear saturation phenomenon. We investigate the spectrum of $n=0$ eigenmodes of the Alfvén wave for cylindrical and toroidal geometries. For the cylindrical case we derive approximate dispersion relations for different situations depending on the profile of $1 /\left(q^{2} \rho\right)$, where $q$ is the safety factor and $\rho$ the mass density. We verify these results with a numerical calculation using the toroidal code LION [11]. We then consider the correlations with the edge plasma density 
in performing a parametric study for a wide range of density profiles. Finally, we consider the possibility to excite the $n=0$ modes with the saddle coils in the JET tokamak.

\section{Toroidal and cylindrical models}

We consider a toroidal axisymmetric plasma in an equilibrium configuration. The electromagnetic oscillations are described by the variational form

$$
\begin{gathered}
\int_{V_{p}}\left\{\left|\nabla \times \vec{E}-\vec{J} E_{b}\right|^{2}-2 \vec{J} \cdot \nabla_{\|} \vec{e}_{n}\left|E_{b}\right|^{2}-\frac{\omega^{2}}{c^{2}} \vec{E}^{\star} \cdot\left(\begin{array}{cc}
\epsilon_{n n} & \epsilon_{n b} \\
\epsilon_{b n} & \epsilon_{b b}
\end{array}\right) \cdot \vec{E}\right\} d^{3} x \\
+\int_{V_{v}}|\nabla \times \vec{E}|^{2} d^{3} x=0
\end{gathered}
$$

where $\vec{J}=\left(\mu_{0} \overrightarrow{j_{0}} \times \overrightarrow{e_{n}}\right) / B_{0}, \overrightarrow{j_{0}}$ is the equilibrium current, $V_{p}$ and $V_{v}$ are the plasma and vacuum volumes and

$$
\begin{aligned}
\epsilon_{n n}=\epsilon_{b b} & =\frac{c^{2}}{v_{A}^{2}} \sum_{i} \frac{f_{i}}{1-\left(\omega / \omega_{c i}\right)^{2}}, \\
\epsilon_{n b}=\epsilon_{b n}^{\star} & =i \frac{c^{2}}{v_{A}^{2}} \sum_{i} \frac{f_{i}\left(\omega / \omega_{c i}\right)}{1-\left(\omega / \omega_{c i}\right)^{2}}, \\
f_{i}=n_{i} m_{i} /\left(\sum_{j} n_{j} m j\right) . &
\end{aligned}
$$

The plasma is surrounded by a vacuum region $V_{v}$ enclosed by a perfectly conducting wall. This model includes finite $\omega / \omega_{c i}$, and, in the limit of $\omega / \omega_{c i} \rightarrow 0$ is equivalent to linearized full ideal MHD in the approximation of $\gamma P \rightarrow 0$, where $\gamma$ is the adiabaticity index and $P$ is the plasma pressure. There are no limiting assumptions on the ratio of the poloidal to the toroidal components of the magnetic field. There are no geometrical expansions or simplifications. The wavefields solution of the weak form is obtained with the LION code using a finite element discretization scheme. This scheme has proven to be numerically converging and pollution- free [11], [12].

In order to obtain approximate dispersion relations with analytical means we model the toroidal axisymmetric plasma of major radius $R$ by a cylindrical, circular, currentcarrying plasma column of periodicity $2 \pi R$. We then define the safety factor $q$ as the inverse rotational transform of the magnetic field over a length of $2 \pi R: q=r B_{z} / R B_{\theta}$. Note that in general both $B_{z}$ and $B_{\theta}$ are functions of the radial coordinate $r$. The equations for the low frequency oscillations in the limit of $\omega / \omega_{c i} \rightarrow 0$ can be written as 


$$
\begin{aligned}
& \frac{d}{d r}\left[\frac{A B^{2}}{A-k_{b}^{2}} \frac{1}{r} \frac{d}{d r}\left(r \xi_{r}\right)\right] \\
& \quad+\left[A B^{2}-\frac{r}{R^{2}} \frac{d}{d r}\left(\frac{B_{z}^{2}}{q^{2}}\right)-\frac{4 n^{2} B_{z}^{2}}{R^{4} q^{2}\left(A-k_{b}^{2}\right)}+r \frac{d}{d r}\left(\frac{2 n k_{b} B B_{z}}{R^{2} q r\left(A-k_{b}^{2}\right)}\right)\right] \xi_{r}=0
\end{aligned}
$$

with

$$
\begin{gathered}
k_{\|}=\frac{B_{z}}{B R}\left(n+\frac{m}{q}\right) \\
k_{b}=\frac{B_{z}}{B}\left(\frac{m}{r}-\frac{n r}{q R^{2}}\right) \\
A=\frac{\omega^{2}-\omega_{A}^{2}}{v_{A}^{2}} \quad \omega_{A}^{2}=v_{A}^{2} k_{\|}^{2} \quad v_{A}^{2}=\frac{B^{2}}{\mu_{0} \rho}
\end{gathered}
$$

where $n$ and $m$ are the toroidal and poloidal mode numbers, $\xi_{r}$ is the plasma radial displacement and $\rho=\rho(r)$ is the mass density. Equation (3) is equivalent to Eq.(1) in the limit of large aspect ratio and small $\omega / \omega_{c i}$. It is important to point out that no approximation on the smallness of $B_{\theta} / B_{z}$ has been made in the derivation of Eqs.(1) and (3). An expansion to the first order in $B_{\theta} / B_{z}$ (see e.g. [13]) results in an inappropriate model for the range of parameters that we are considering in this paper. Indeed, such a model predicts for $n=0$, in the limit $\omega / \omega_{c i}=0$, the existence of a GAE in shearless plasmas, whereas it is easy to show that the exact model predicts no global eigenmode in this case [14].

Equation (3) describes two types of waves, the fast magnetosonic wave and the Alfvén wave. The latter has a spectrum including two types of eigenmodes: a continuum and a discrete set of eigenmodes called the Global Alfvén Eigenmodes (GAE). The existence of GAEs can be demonstrated by carrying out a WKB analysis of Eq.(3). We obtain

$$
\omega^{2}=\omega_{A}^{2}-\frac{1}{\mu_{0} \rho k_{r}^{2}}\left[\left(\frac{2 B_{z} n}{q R^{2}}\right)^{2}-k_{b}^{2} \frac{r}{R^{2}} \frac{d}{d r}\left(\frac{B_{z}}{q^{2}}\right)\right]
$$

For $n=0$ we have then

$$
k_{r}^{2}=\frac{\left(-1 / \mu_{0} \rho\right)}{\omega_{A}^{2}-\omega^{2}} \frac{B_{z}^{2}}{B^{2}} \frac{m^{2}}{r} \frac{1}{R^{2}} \frac{d}{d r}\left(\frac{B_{z}}{q^{2}}\right)
$$


For a plasma of finite size (radius $a$ ) the quantization condition $\int_{0}^{a} k_{r} d r=l \pi$ gives a discrete spectrum of eigenfrequencies of the Alfvén wave.

From Eq.(8) we can see that the mode existence and localization in space depends crucially on the $q$ and $B_{z}$ profiles. For tokamaks, $B_{z}(r)$ is nearly constant and we have $k_{r}^{2}>0$ if $d q / d r>0$ and $\omega<\omega_{A}$. This means that the spectrum of $n=0$ GAEs, if they exist, is Sturmian for positive shear, with eigenfrequencies below the continuum frequencies. If $d q / d r<0$, we have $k_{r}^{2}>0$ for $\omega<\omega_{A}$, which means that in reversed shear tokamaks the spectrum of $n=0$ GAEs is anti-Sturmian with eigenfrequencies above the continuum frequencies. In the absence of shear there are no $n=0$ GAEs.

We note also that $k_{r}^{2}$ is large where $|d q / d r|$ is large and where $\left|\omega_{A}-\omega\right|$ is small. This implies a mode localization near the edge for typical standard tokamak $q$ profiles that peak near the edge. This mode localization will be pronounced if the profile of $\omega_{A}(r)$ is monotonically decreasing with $\mathrm{r}$, because $\omega_{A}-\omega$ will be smallest near the edge.

The $n=0$ continuum can be written as

$$
\omega_{A}^{2}(r)=\frac{B_{z}^{2}(r) m^{2}}{\mu_{0} \rho(r) q^{2}(r) R^{2}}
$$

We note that the continuum frequencies are proportional to $|m|$. Thus there are no crossings of continua for different $m$. Therefore toroidal coupling cannot create gaps in the continuum and there are no $n=0$ TAEs.

The $n=0$ case is characterized by an invariance with respect to the sign of $m$, as can be seen by inspecting Eq.(3). This means that for any given eigenfrequency the eigensolution is a superposition of positive and negative $m s$, with relative amplitudes and phases determined by the boundary conditions. This can explain why the "AFM" seen in TFTR experiments has a poloidally standing wave structure and why the mode is "antiballooning" when the plasma is touching the inner limiter, whereas it is "ballooning" when the plasma is touching the outer limiter [1].

\section{Analysis of $n=0$ GAEs in cylinder}

Let us now analyze Eq.(8) in different cases.

(I) Case $\omega_{A}^{2}(r)=$ const 
Defining the frequency separation between the GAE and the continuum as

$$
\Delta=\frac{\omega_{A}^{2}-\omega^{2}}{\omega_{A}^{2}}
$$

we can write

$$
k_{r}^{2}=\frac{1}{\Delta} \frac{B_{z}^{2}}{B^{2}} \frac{1}{r}\left(-\frac{d}{d r} \ln \rho\right)
$$

We note that this expression does not depend on $m$. It can thus be expected that the Alfvén spectrum will be replicated at multiple $m$ values. For a "tokamak" case $\left(B_{z}=\right.$ const $)$ we have $k_{r}^{2}=(1 / \Delta)(2 / r)(d \ln q / d r)$. Let us consider a concrete example. For density profiles parametrized with

$$
\rho=\left(1-p(r / a)^{2}\right)^{\kappa}
$$

where $p$ and $\kappa$ are given constants we obtain

$$
\Delta=2 \kappa\left(\frac{\arcsin (\sqrt{p})}{l \pi}\right)
$$

For $\kappa>0$ the spectrum of $n=0$ GAEs is Sturmian. There is an infinity of modes with an accumulation point at $\omega=\omega_{A}$. The separation between eigenfrequencies is proportional to $1 / l^{2}$. For $p=1$ we have checked the prediction of the WKB approximation, which gives $\Delta=50 \%$, against a global calculation with the LION code, which gives $\Delta=44.2 \%$, for the first radial eigenmode. The agreement can be considered as sufficiently good considering that the radial wavelength of this GAE is of the same order as the plasma size. The second radial eigenmode has $\Delta=12.5 \%$ from the WKB analysis and $\Delta=11.1 \%$ from the LION code. Fig.1 shows the two first $m=-1$ radial eigenmodes as calculated with the LION code. In this calculation the wall was placed right on the plasma boundary.

(II) Case $\omega_{A}^{2}(r)$ has a local extremum at $r=r_{c} \neq 0$

This means that

$$
\left.\frac{d}{d r}\left(\frac{B_{z}^{2}}{q^{2} \rho}\right)\right|_{r=r c}=0
$$

A local analysis of Eq.(3) can be made in a similar way as in Ref.[15]. Defining $x=r-r_{c}$ and looking for solutions of the type $\xi_{r}=x^{\alpha}$ the characteristic equation can be written as

$$
\alpha^{2}+\alpha-g=0, \quad g=-\frac{2}{r} \frac{B_{z}^{2}}{B^{2}} \frac{d(\ln \rho) / d r}{d^{2}\left(\ln \omega_{A}^{2}\right) / d r^{2}}
$$


For "tokamak" case $\left(B_{z} \approx B \approx\right.$ const $)$ the last term in Eq.(15) can be written as

$$
g=\frac{4}{r} \frac{B_{z}^{2}}{B^{2}} \frac{d(\ln q) / d r}{d^{2}\left(\ln \omega_{A}^{2}\right) / d r^{2}}
$$

If the discriminant of Eq.(15) is negative then there exists an oscillatory solution in the vicinity of $r=r_{c}$ and the extremum of the continuum is an accumulation point of a discrete spectrum. The sufficient condition for this is $g>1 / 4$. We verify again that the crucial parameter for the existence of $n=0 \mathrm{GAEs}$ in tokamaks is the shear. For positive shear, $n=0$ GAEs can exist below a local minimum of the continuum, while for negative shear $n=0$ GAEs can exist above a local maximum of the continuum, provided that the shear is large enough in absolute value. We note also that, as in the WKB analysis, the existence condition does not depend on the poloidal mode number $m$.

(III) Case $\omega_{A}^{2}(r)$ has a local extremum at $r=0$

This means that

$$
\left.\frac{d}{d r}\left(\frac{B_{z}^{2}}{q^{2} \rho}\right)\right|_{r=0}=0
$$

The same local analysis of Eq.(3) as for case(II) can be made. In this case the characteristic equation is

$$
r^{2} \xi_{r}^{\prime \prime}+5 r \xi_{r}^{\prime}+\left(3-m^{2}+g\right) \xi_{r}=0
$$

The condition for the existence of oscillatory solutions is then $g>m^{2}+1$.

(IV) Case $\omega_{A}^{2}(r)$ is monotonic

This means that

$$
\frac{d}{d r}\left(\frac{B_{z}^{2}}{q^{2} \rho}\right)\left\{\begin{array}{l}
<0 \\
>0
\end{array}\right.
$$

Let us look for eigenfrequencies in the continuum. For $\omega$ in the range of continuum frequencies, the wavefield has a singularity at a position $r=r_{s}$. Global modes that may eventually exist in this continuum range are subject to resonant absorption, a process sometimes called continuum damping. In this case the spectrum can be represented by complex eigenfrequencies (this is the so-called "quasi-mode" representation). We shall first concentrate on the real part of the eigenfrequency, and consider the imaginary part at a later stage when the equations are solved numerically.

Although the WKB approximation cannot be used in a part of the plasma $\left(r>r_{s}\right.$ or $r<r_{s}$, depending on the sign of $\left.\left(\omega_{A}^{2}\right)^{\prime}(r)\right)$ we shall see that it can still give qualitatively 
correct results. Let us first rewrite the expression for $k_{r}^{2}$ given by Eq.(8):

$$
k_{r}^{2}=-\frac{B_{z}^{2}}{B^{2}} \frac{1}{r} \frac{1}{\Delta}\left(\frac{d}{d r} \ln \left(\omega_{A}^{2}\right)+\frac{d}{d r} \ln \rho\right)
$$

For "tokamak" case $\left(B_{z} \approx B \approx\right.$ const $)$ this reads

$$
k_{r}^{2}=\frac{2}{r} \frac{1}{\Delta} \frac{d}{d r} \ln q
$$

The radial wavenumber goes to infinity at the local Alfvén resonance $r=r_{s}$. The quantization condition

$$
\begin{array}{ll}
\int_{0}^{r_{s}} k_{r} d r=l \pi & \text { if }\left(\omega_{A}^{2}\right)^{\prime}(r)<0 \\
\int_{r_{s}}^{1} k_{r} d r=l \pi & \text { if }\left(\omega_{A}^{2}\right)^{\prime}(r)>0
\end{array}
$$

where $l$ is an integer, is nevertheless integrable if we assume a local linear dependence of $\omega_{A}^{2}(r)$ in the vicinity of $r=r_{s}$. The exact resolution of Eq.(22) is practically inconvenient since the integration boundary $r_{s}$ depends on the frequency $\omega$, which is given by the quantization condition. Both $r_{s}$ and $k_{r}$ depend on the actual profile of $\omega_{A}^{2}(r)$. To show the qualitative behaviour of these "global quasi-modes" we consider a case $B_{z} \approx B \approx$ const with a $q$ profile given by

$$
q=q_{0}\left(1+b(r / a)^{2}\right)^{\kappa_{q}}
$$

and approximate $\Delta$ in the vicinity of the singular point $r=r_{s}$ as

$$
\Delta \approx\left(r_{s}-r\right) / \alpha
$$

Let us look for solutions with $r_{s}$ near the plasma edge. In this case the quantization condition gives approximately

$$
\alpha \frac{r_{s}}{a}=\frac{l^{2} \pi^{2}}{16 \kappa_{q} b}
$$

Note that there can be more than one solution for a given "radial mode number" $l$. The quantity $\alpha$ is inversely prorportional to the derivative of $\omega_{A}^{2}$ at $r=r_{s}$. So $\alpha$, in general, depends on $\omega$. So does the resonance position $r_{s}$. Therefore $\mathrm{Eq}(25)$ can have multiple solutions. If $\omega_{A}^{2}(r)$ has a parabolic profile, for example, $\alpha$ can be approximated with a linear function of $\omega$. At lowest order $r_{s}$ is also linear in $\omega$. Therefore it can be expected that in such cases there are two eigenfrequencies for each radial mode number $l$. 


\section{Global wave results in torus}

We note that the $n=0$ GAE exists even with a wall right on the plasma boundary (see Fig.1). This mode, although global in its wavefield extension, is not an "external" mode. We have checked with the LION code the influence of the plasma-wall distance on the mode frequency. With a wall far away from the plasma, the eigenfrequency is only $3.3 \%$ lower than with a wall right on the plasma boundary.

The second point to check is the importance of toroidal effects. We have made an aspect ratio study and found that the eigenfrequency is quite insensitive. From an aspect ratio of 300 down to 3 , the eigenfrequency moves up by less than $3 \%$. No other $n=0$ mode appears due to toroidicity. This confirms the analysis developed in the previous section: the mode is not toroidicity-induced. Toroidicity nevertheless affects the eigenmode structure. In Fig.2 we show the poloidal Fourier decomposition of the first radial " $m=-1$ " mode for a plasma of aspect ratio 3 and a wall/plasma radius ratio of 1.1 (the other parameters are the same as for Fig.1). There is a substantial amount of poloidal mode numbers $m=0,1,-2$ in the wavefield, due to the coupling by toroidicity from the $m=-1$ antenna excitation in this case.

Let us now examine the eigenfrequency behaviour with respect to the density profile. In TFTR it has been observed that the eigenfrequency of the AFM follows closely the Alfvén continuum frequency near the plasma edge. To demonstrate that the $n=0 \mathrm{GAE}$ also follows the same behaviour, we consider a circular plasma of aspect ratio 3 , with a $q$ profile going from $q_{0}=0.88$ to $q_{a}=3.2$. In the experiment, the density profile has been varied by pellet injection or strong gas puffing on a timescale short enough that we can assume the $q$ profile to have remained the same. In our calculations we do not want to examine closely a specific shot but we rather choose a sequence of density profiles that reproduces the essential features of the experimental ones, and keep the same $q$ profile. The density profiles are given by Eq.(12) with $p=0.9$ and $\kappa$ varying from 0.1 to 1.7 . The corresponding density, $q$ and $\omega_{A}$ profiles are shown in Fig. 3. For each value of $\kappa$ we compute the eigenfrequencies with the LION code. The results are shown in Fig.4, where these frequencies are plotted as a function of the continuum frequency at $90 \%$ of the plasma minor radius, $\omega_{A}(.9)$. The frequencies are normalized to the Alfvén transit time $R_{0} / v_{A 0}$, where $R_{0}$ is the radius of magnetic axis and $v_{A 0}$ is the Alfvén velocity on magnetic 
axis. (For typical TFTR parameters $R_{0}=2.52 m, B_{0}=5 T, n_{e 0}=7.0 \times 10^{19} m-3$, deuterium plasma, a normalized frequency of 1 corresponds to a frequency of $520 \mathrm{kHz}$ ). The horizontal dotted line corresponds to the half the core continuum Alfvén frequency, $\omega_{A}(0) / 2$. (This is for comparison with Fig.5 of Ref. [1]; the experimental frequency range of observation corresponds roughly to $\omega=0.4-0.7$ ). The minimum continuum frequency is marked by the dash-dotted line in Fig. 4. Our results show clearly that in the range $\omega=0.4-0.7$ the $n=0$ eigenfrequency follows closely the edge Alfvén frequency $\omega_{A}(.9)$ and not the core Alfvén frequency, in excellent agreement with experimental observations reported in Ref.[1].

Fig. 4 shows the first two radial $m=1$ eigenmodes of the $n=0$ GAE. The two branches are frequency separated by about $25 \%$. As the eigenfrequency enters into the continuum, each branch splits into two modes, as expected from the analytical theory presented in Section 3, case IV. From the response given by the LION code, we can measure the continuum damping of these modes: $\gamma / \omega$ is typically of the order of a few percent. The Alfvén resonance surface is, for the case of Fig. 4, between $r / a=0.9$ and $r / a=1$. As $\omega_{A}(.9)$ and the eigenfrequency decrease the continuum damping increases and the resonance surface moves deeper into the plasma.

We show in Fig. 5 the wavefields of the first radial mode of the $n=0, m=-1$ GAE for the case $\kappa=0.4, \omega_{A}(.9)=.545, \omega=0.536$. The radial electric field component, $E_{n}$, is quite edge-localized, whereas the binormal electric field component, $E_{b}$, is very global. In fact, the $n=0 \mathrm{GAE}$ is a finite frequency version of an internal kink mode.

There are other eigenmodes in the system. One important mode is the surface mode of the fast magnetosonic wave [16]. For the same case as in Fig.5, we show in Fig.6 the wavefields of the $m=-1$ surface mode which is found at $\omega=1.28$. It has a $m=-2$ resonance surface at $s=0.86$. This mode is evanescent everywhere and therefore cannot be found with the WKB analysis presented in Section3. The $n=0$ surface mode is a finite frequency version of an external kink mode.

At frequencies comparable to the surface mode, we find also the $m=-2 n=0$ GAEs. For the case $\kappa=0.7, \omega_{A}(.9)=0.662$, the first radial mode is found at $\omega=1.43$. We show in Fig. 7 the corresponding wavefields. Besides a different poloidal composition this modes resembles the $m=-1 n=0$ GAE. Note that the $m=-1$ first radial mode has an eigenfrequency $\omega=0.653$, which is close to a factor 2 smaller. In fact, we have found 
that the $|m|=2$ spectrum is nearly similar to the $|m|=1$ spectrum, thereby confirming the analysis of Section 3. The factor of about 2 is also confirmed experimentally: in Fig.11 of Ref.[1], the measurements show the "higher frequency AFM branch at the end of the discharge" at a factor 2 above the lower branch. For this case, we can interpret the two branches as $|m|=1$ and $|m|=2 n=0$ GAEs.

In other situations a smaller difference (typically $25 \%$ ) in frequency is observed between the two branches (see e.g. Fig.2 of Ref.[1]). In view of our results (Fig.4), we can interpret these two branches as the first two radial modes of the $|m|=1 n=0$ GAE.

\section{Excitation of $\mathbf{n}=0$ GAEs in JET}

We now consider a typical JET equilibrium in a single null configuration of aspect ratio 2.8 , elongation 1.7, with $q_{0}=1.2, I=3 M A, R_{0}=2.9 m, B_{0}=3.4 T, n_{e 0}=3 \times$ $10^{19} \mathrm{~m}^{-3}$, deuterium plasma. The saddle coils installed in the JET vacuum vessel, if phased properly, could be used to excite $n=0$ modes.

We show in Fig. 8 the result of computations made with the LION code. The antenna resistive power is plotted versus the applied frequency. The case of $n=0$ phasing of the saddle coils is marked in continuous line and the case of $n=2$ phasing is marked in dashed line for the sake of comparison. The type of modes $(\mathrm{G}=\mathrm{GAE}, \mathrm{T}=\mathrm{TAE}, \mathrm{E}=$ $\mathrm{EAE}, \mathrm{S}=$ surface mode) is identified above the corresponding peak in the figure. With $n=0$ phasing the antenna excite successively the first radial eigenmode of the $|m|=1$ $\mathrm{GAE}$, the second radial eigenmode of the $|m|=1 \mathrm{GAE}$, the $|m|=1$ surface mode (with a large continuum damping) and the first radial eigenmode of the $|m|=2 \mathrm{GAE}$. We point out that this classification of modes according to the poloidal mode number $m$ is made by analogy with the cylindrical case and does not necessarily represent the poloidal mode behaviour correctly: the poloidal Fourier decomposition of the modes for the JET geometry is even broader than that for the circular case of previous section.

Thus we predict that the excitation of $n=0$ GAEs is feasible with the existing JET saddle coils. As can be seen in Fig. 8, the $n=0,|m|=1$ GAE frequency lies between the TAE and EAE frequencies. This could be deduced from the fact that the $n=0$ GAE frequency is below (or near the lower edge) of the $n=0$ Alfvén continuum given by Eq.(9). For $|m|=1$ it is exactly at twice the frequency of the center of the TAE gap 
or, equivalently, at the frequency of the center of the EAE gap.

\section{Conclusion}

We have presented a theory, both analytical and numerical, of the $n=0$ GAEs. In terms of eigenfrequencies and their behaviour with plasma parameters such as the density profile, we have provided an explanation for the observation of the "AFM" mode. All the characteristics of this mode as enumerated in Section 1 have been examined and can be explained by the present theory. In addition, we have made a prediction of the possible excitation of these modes by the saddle coil antennas in the JET experiment.

The question of the spontaneous excitation of the $n=0 \mathrm{GAE}$ is a more challenging issue. We have not addressed it in this paper but would like to point out several unresolved facts that future theoretical works in the field of Alfvén mode stability should tackle. Why is the "AFM" ( $n=0 \mathrm{GAE})$ nearly always present in the experiment but not the TAE? If a charge exchange mechanism is responsible for the excitation of the $n=0$ GAE, the same mechanism should act in the same way on TAEs. Why is the $n=0 \mathrm{GAE}$ apparently insensitive to the presence of fast particles? We have shown that this mode, although correlated with the edge density, is very global and not so much edge-localized. It is basically the same wave (Alfvén) as the TAE, in the same range of frequency. The same damping and driving mechanisms should act similarly on the $n=0 \mathrm{GAE}$ and the TAE. Theoretical predictions of TAE stability should be accompanied by predictions of $n=0$ GAE stability under the same assumptions.

\section{Acknowledgments}

This work was partly supported by the Swiss National Science Foundation. 


\section{References}

[1] CHANG, Z., et al., Nucl. Fusion 35 (1995) 1469.

[2] ROSENBLUTH, M.N. and RUTHERFORD, P.H., Phys. Rev. Lett. 34 (1975) 1428.

[3] MIKHAILOVSKII, A.B., Sov. J. Plasma Phys. 1 (1975) 38.

[4] CHENG, C.Z., CHANCE, M.S., Phys.Fluids 29 (1986) 3695.

[5] WONG, K.L., FONCK, R.J., PAUL, S.F., ET AL., Phys.Rev.Lett. 66 (1991) 1874.

[6] HEIDBRINK, W.W., STRAIT, E.J., DOYLE, E., ET AL., Nucl.Fusion 31 (1991) 1635.

[7] STRAIT, E.J., HEIDBRINK, W.W., TURNBULL, A.D., CHU, M.S., DUONG, H.H., Nucl.Fusion 33 (1993) 1849.

[8] WELLER, A., et al., Phys. Rev. Lett. 72 (1994) 1220.

[9] FASOLI, A., et al., in Plasma Physics and Controlled Nuclear Fusion Research 1994 (Proc. 15th Int. Conf. Seville, 1994), Vol. 1, p.405, IAEA, Vienna, 1995.

[10] FASOLI, A., LISTER, J.B., MORET, J.-M., et al., Phys. Rev. Lett. 75 (1994) 645.

[11] VILLARD, L., APPERT, K., GRUBER, R., VACLAVIK, J., Comput.Phys.Rep. 4 (1986) 95.

[12] VILLARD, L., FU, G.Y., Nucl.Fusion 32 (1992) 1695.

[13] APPERT, K., VACLAVIK, J., Plasma Phys. 25 (1983) 551.

[14] SHAFRANOV, V.D., Sov. Phys. - JETP 15 (1970) 175.

[15] APPERT, K., GRUBER, R., TROYON, F., VACLAVIK, J., Plasma Phys. 24 (1982) 1147.

[16] APPERT, K., VACLAVIK, J., VILLARD, L., Phys. Fluids 27 (1984) 432. 


\section{Figure Captions}

Fig.1. First two radial eigenmodes $(l=1, l=2)$ of the $m=-1, n=0$ GAE in a cylinder. Case $\omega_{A}(s)=$ const. Normal (top) and binormal (bottom) components of the wave electric field.

Fig.2. First radial eigenmode of the $m=-1, n=0$ GAE in a torus of aspect ratio 3 and circular cross-section. The labels mark the dominant poloidal mode numbers $m$. Normal (top) and binormal (bottom) components of the wave electric field.

Fig.3. Profiles of $q$ (top), mass density (middle) and $n=0,|m|=1$ Alfvén continuum frequency (bottom) for different density profiles given by Eq.(12) with $p=0.9$ and various values of $\kappa$ marked in the figure.

Fig.4. Frequencies of the $m=-1, n=0$ GAEs versus continuum frequency at $s=0.9$ in a toroidal plasma of aspect ratio 3 , circular cross-section and various density profiles as in Fig.3. The frequencies are normalized to the Alfvén transit time $R_{0} / v_{A 0}$. ( $\omega=1$ corresponds to $f=520 \mathrm{kHz}$ for typical TFTR parameters $R_{0}=2.52 \mathrm{~m}$, $B_{0}=5 T, n_{e 0}=7 \times 10^{19} \mathrm{~m}^{-3}$, Deuterium).

Fig.5. First radial $m=-1, n=0$ GAE for the same parameters as in Fig.3, with $\kappa=0.4, \omega_{A}(.9)=.545, \omega=.536$. The labels mark the dominant poloidal mode numbers $m$.

Fig.6. Surface mode $m=-1, n=0$ for the same parameters as in Fig.3, with $\kappa=0.4$, $\omega_{A}(.9)=.545, \omega=1.28$. The labels mark the dominant poloidal mode numbers $m$.

Fig.7. First radial $m=-2, n=0$ GAE for the same parameters as in Fig.3, with $\kappa=0.7, \omega_{A}(.9)=.662, \omega=1.43$. The labels mark the dominant poloidal mode numbers $m$.

Fig.8. Resistive power coupled by saddle coils antennas versus applied frequency for two different antenna phasings: $n=0$ (continuous line) and $n=2$ (dashed line). The parameters are: JET single-null configuration, aspect ratio 2.8, elongation 1.7, $q_{0}=1.2, I=3 M A, R_{0}=2.9 m, B_{0}=3.4 T, n_{e 0}=3 \times 10^{19} m^{-3}$, Deuterium plasma. $\mathrm{T}=\mathrm{TAE}, \mathrm{G}=\mathrm{GAE}, \mathrm{S}=$ Surface mode, $\mathrm{E}=\mathrm{EAE}$. 

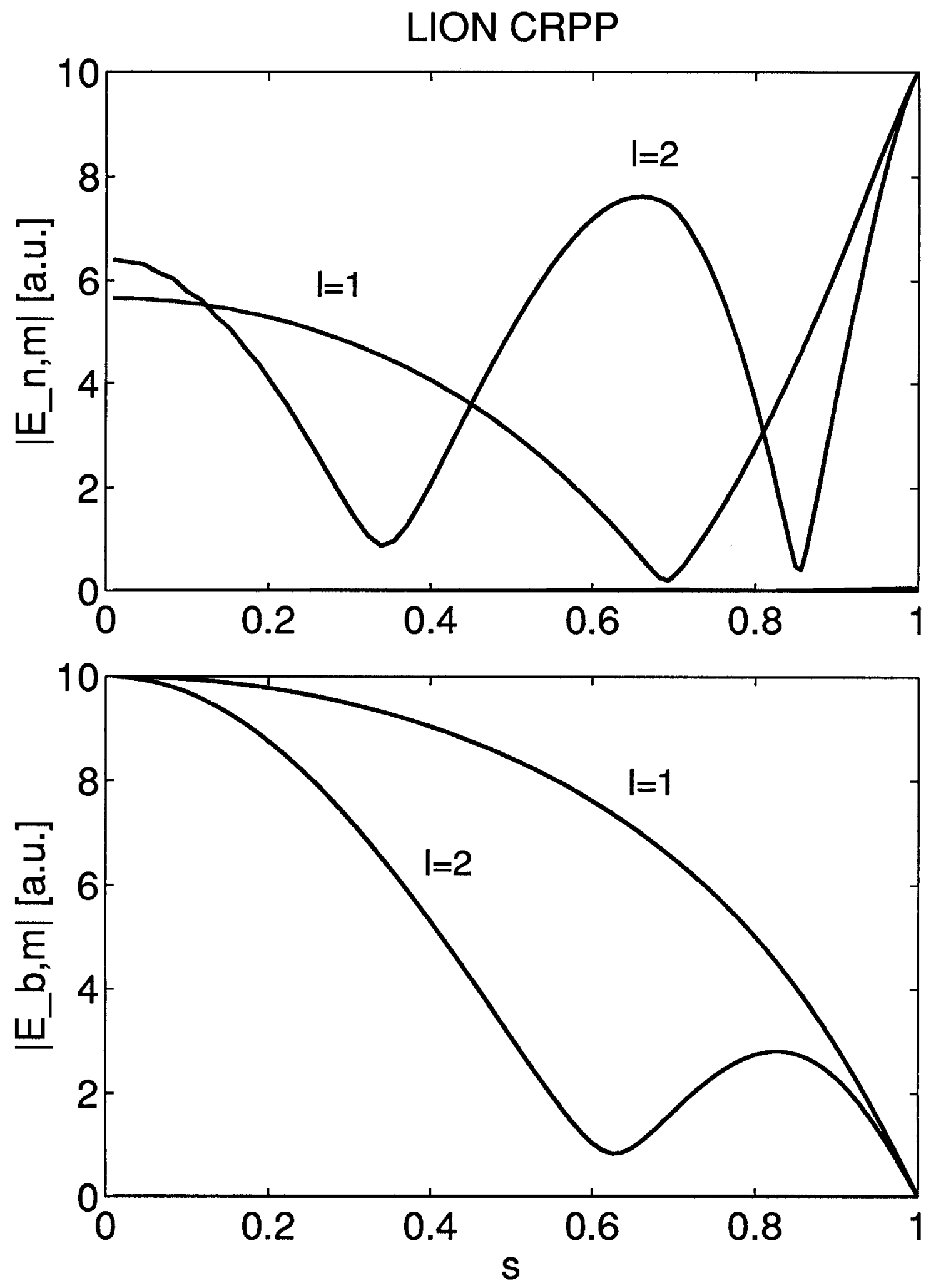

Fig.1 

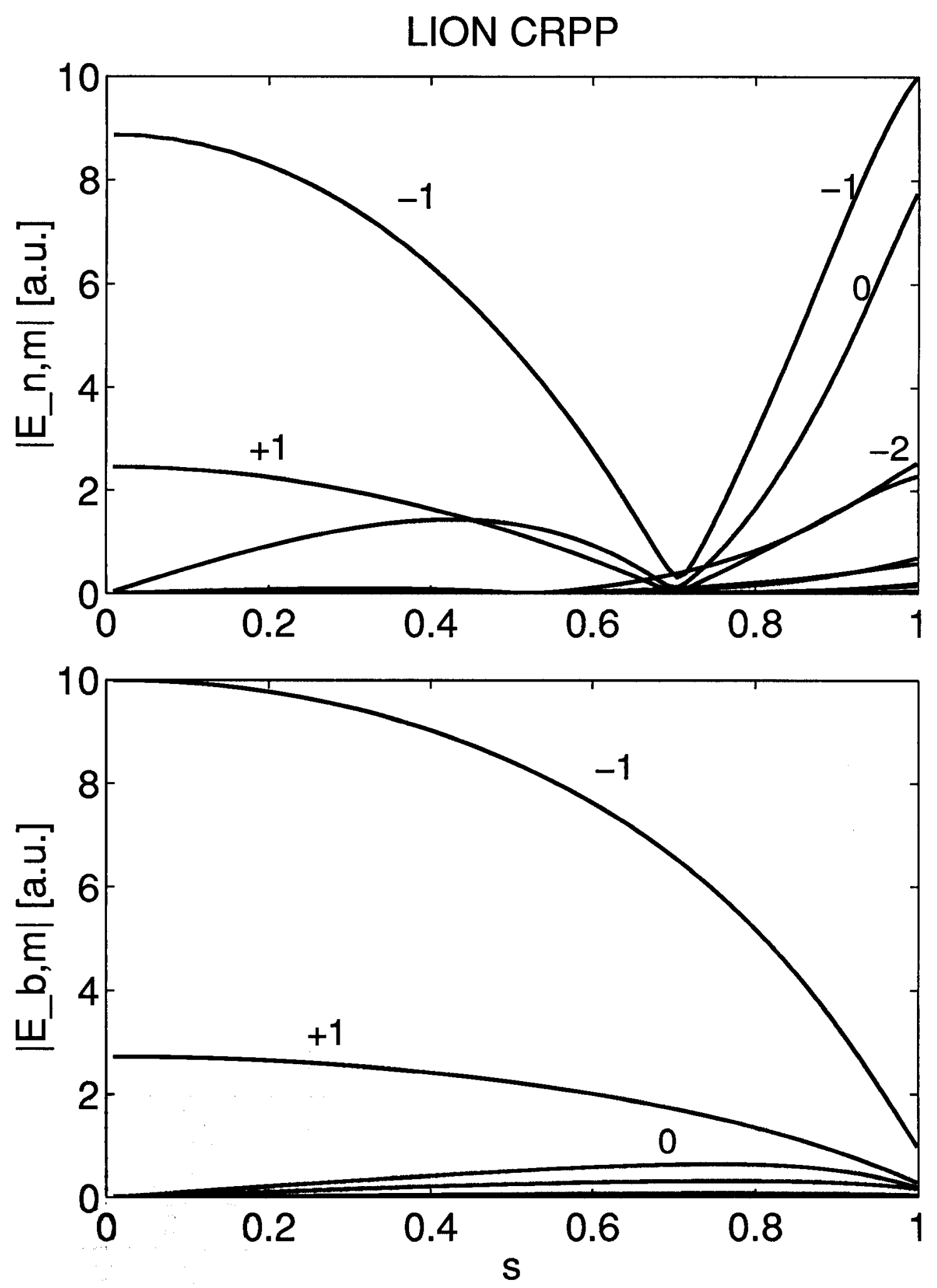

Fig.2 


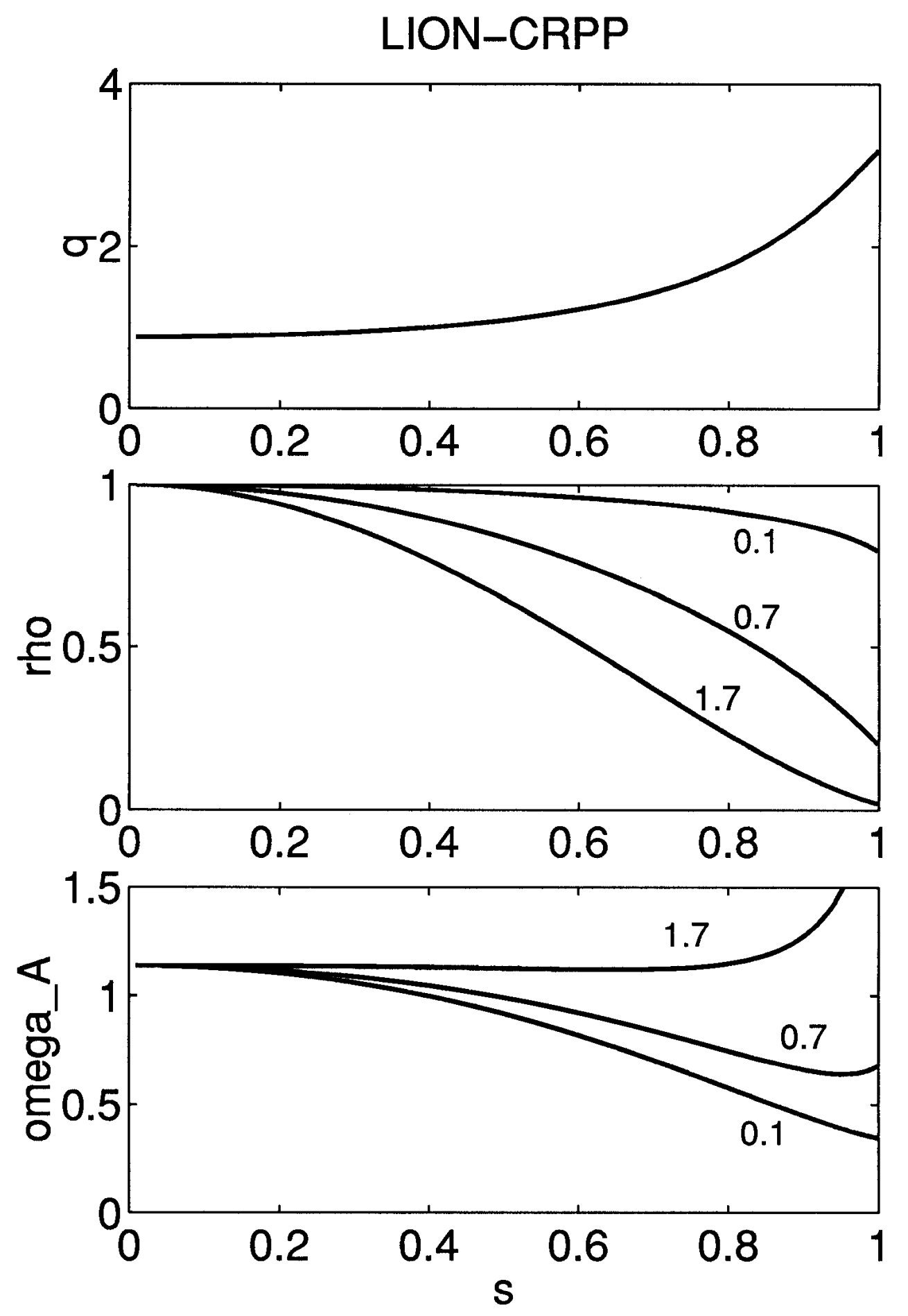

Fig.3 


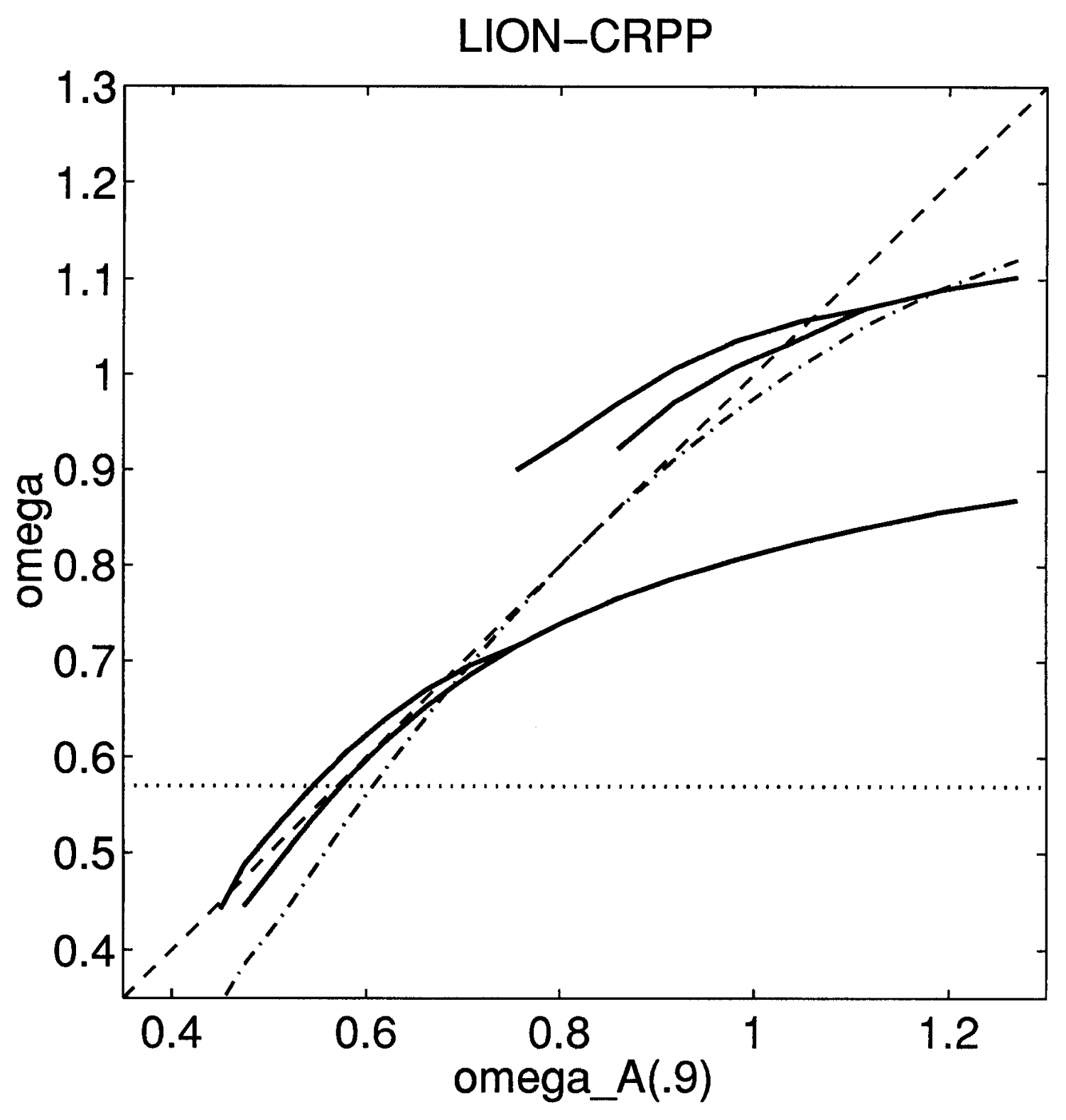

Fig.4 
LION-CRPP
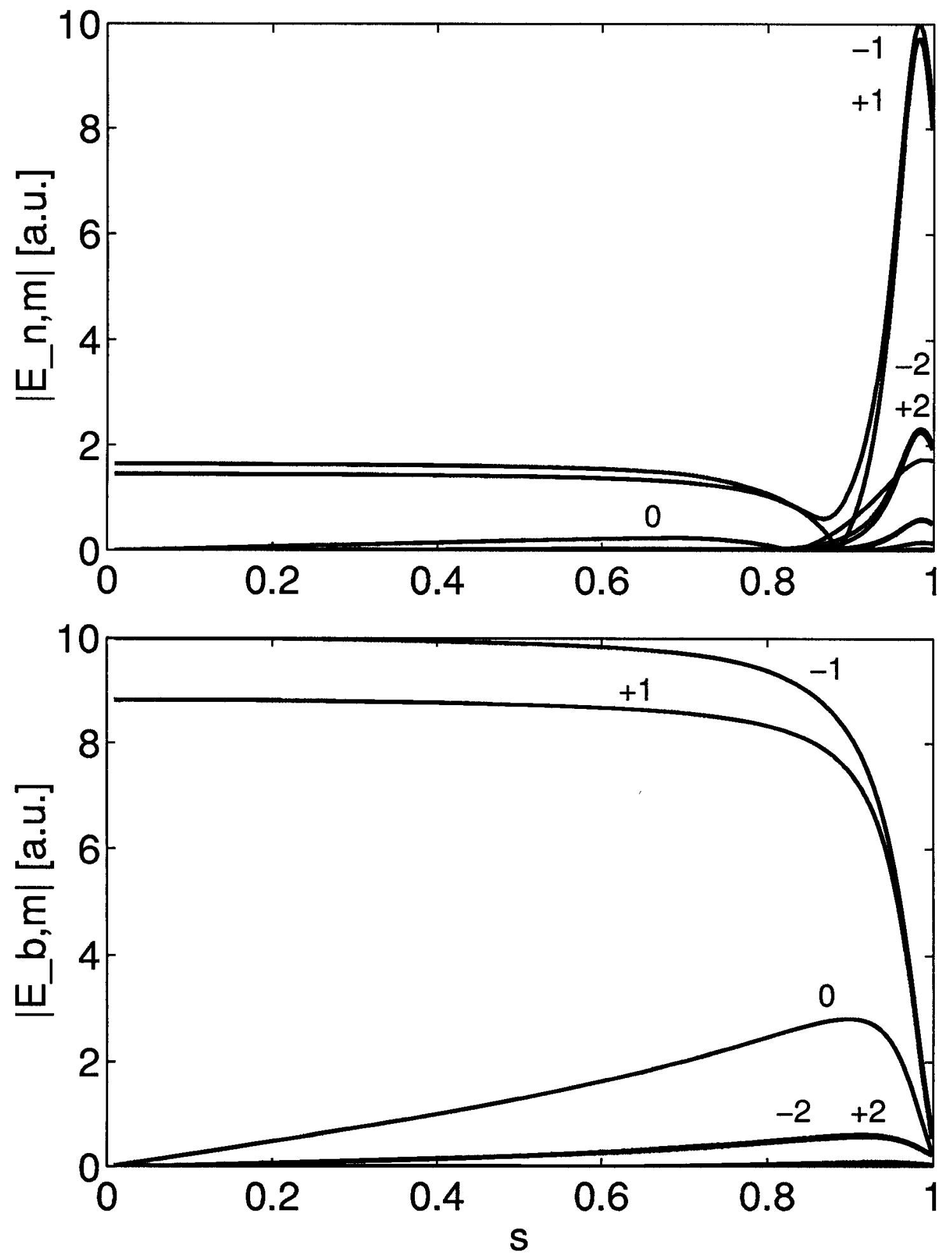

Fig.5 
LION-CRPP
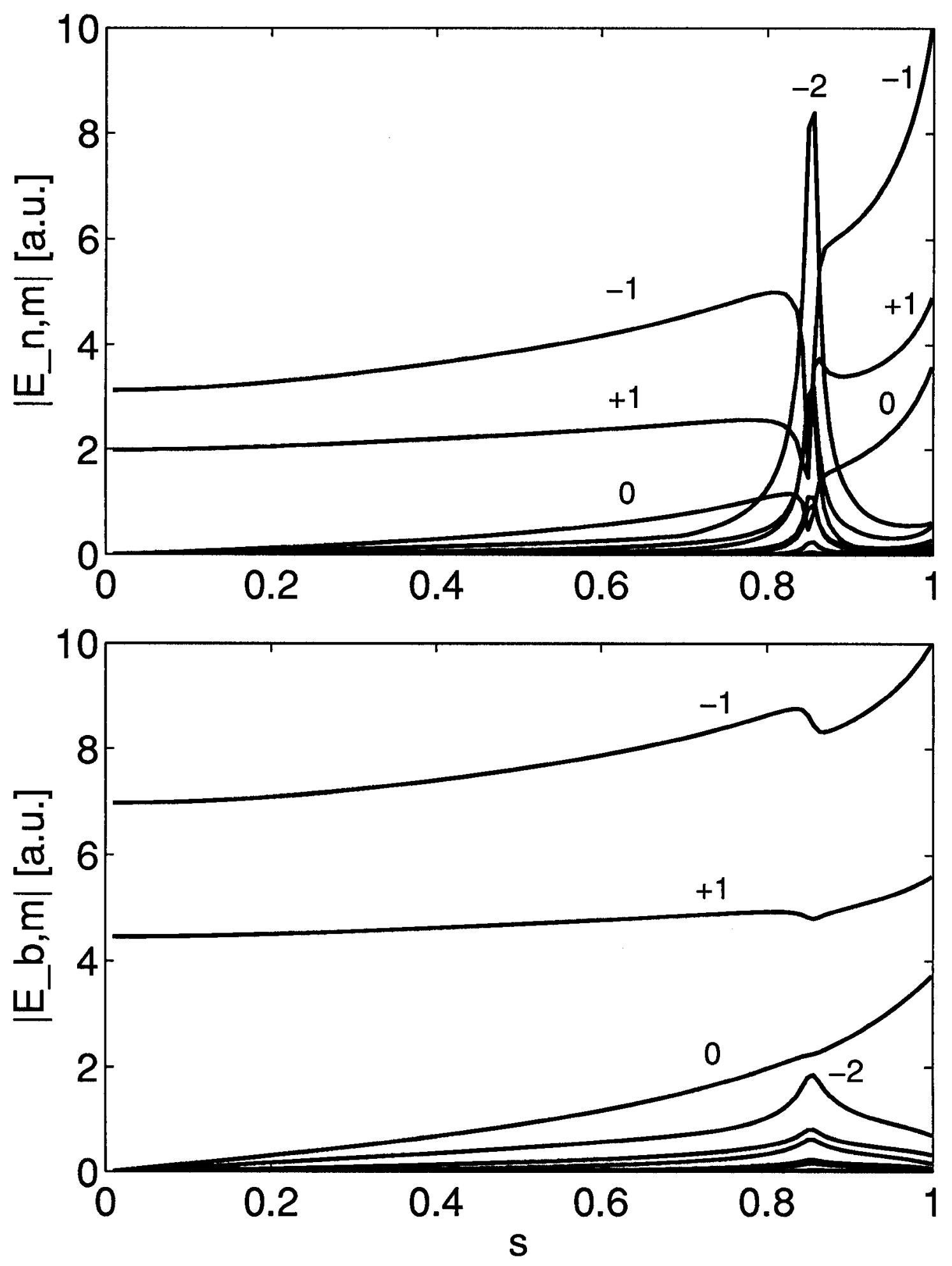

Fig.6 

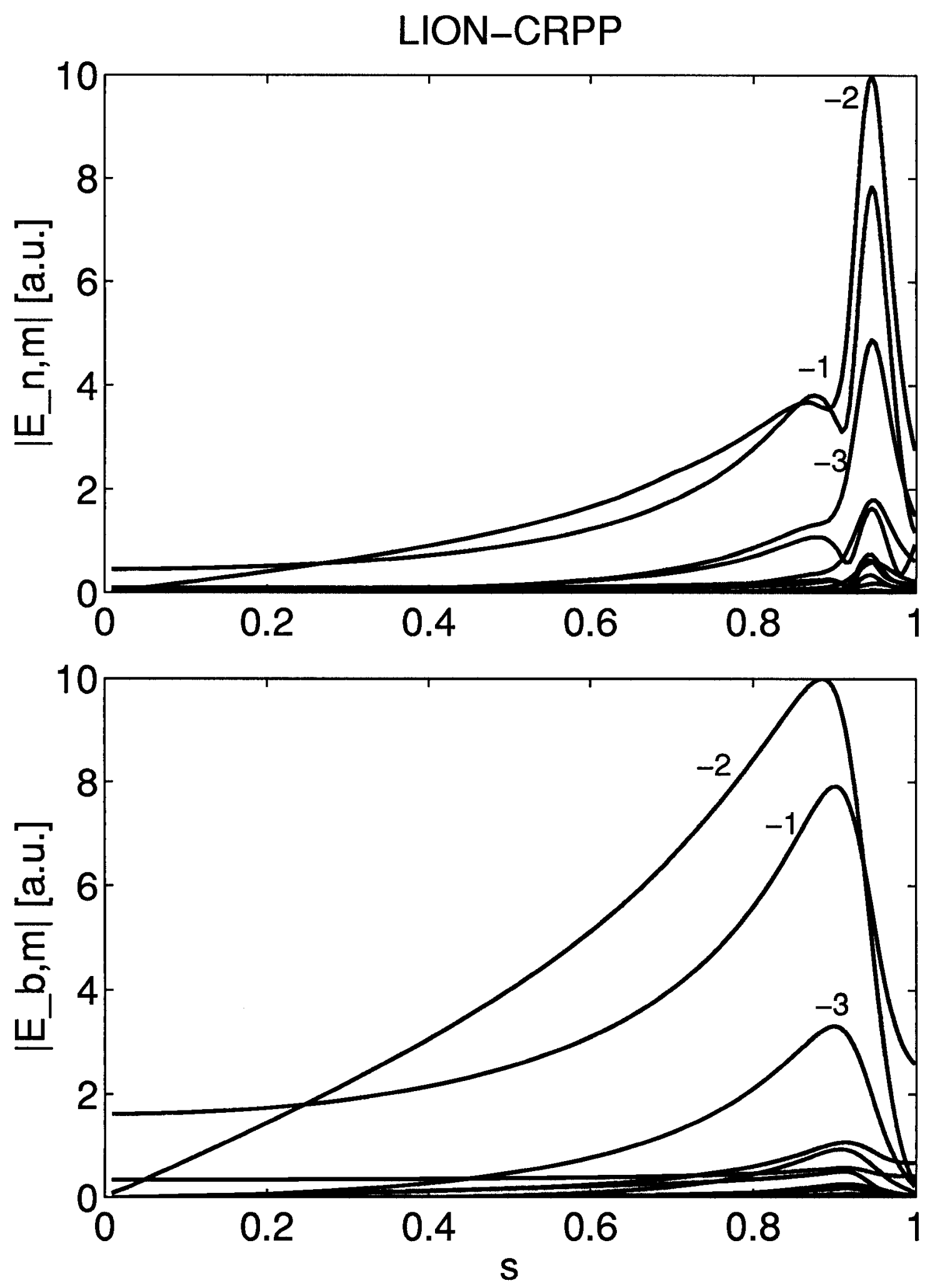

Fig.7 


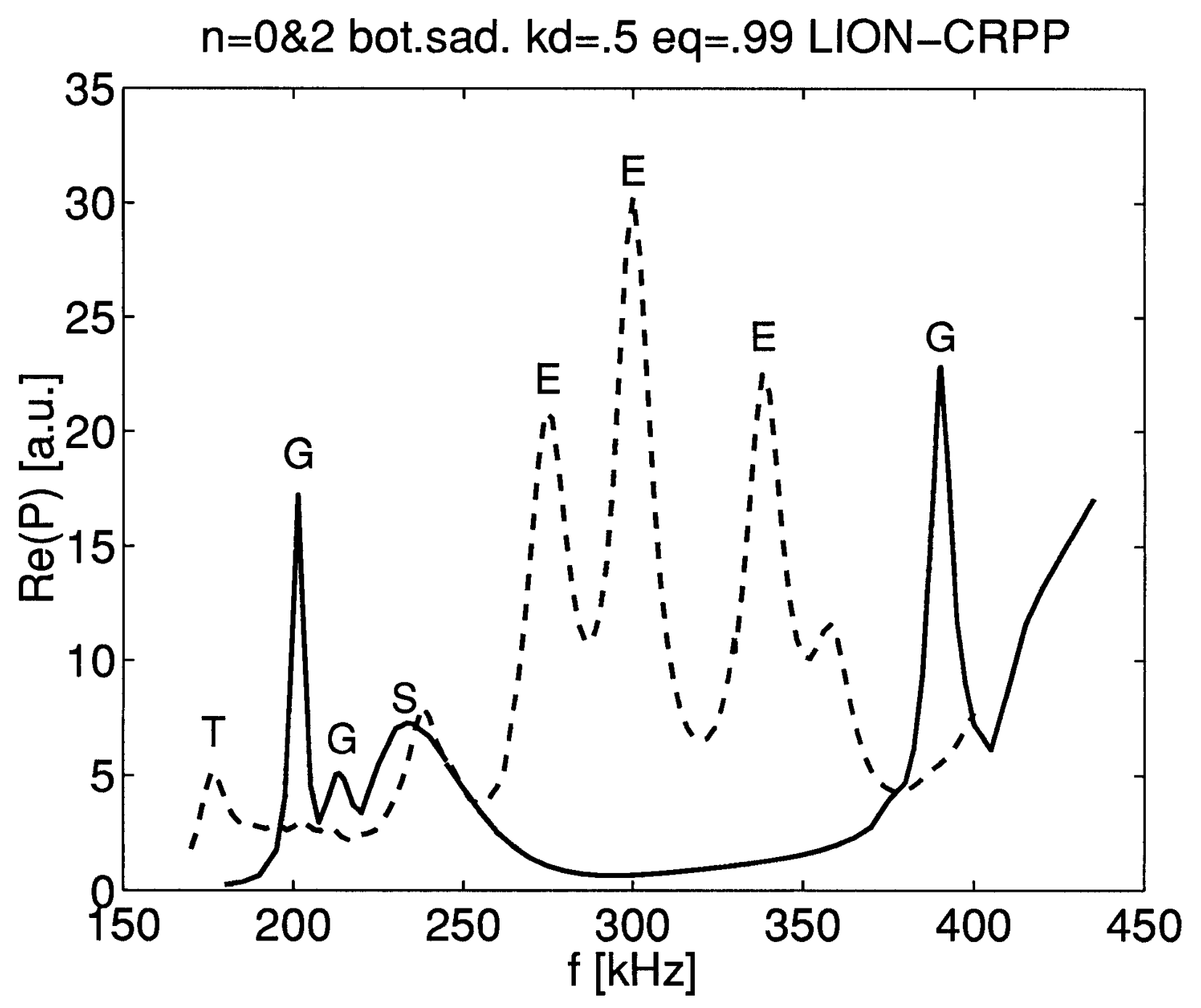

Fig.8 